\title{
Histologic Abnormalities in Children with Nonalcoholic Fatty Liver Disease and Normal or Mildly Elevated Alanine Aminotransferase Levels
}

\author{
Jean $\mathbf{P}$ Molleston, $\mathbf{M D}^{1}$, Jeffrey $\mathbf{B}$ Schwimmer, $\mathbf{M D}^{2}$, Katherine $\mathbf{P}$ Yates ${ }^{3}$, Karen $\mathbf{F}$ Murray, \\ $M^{4}$, Oscar W Cummings, $M^{5}$, Joel E. Lavine, MD, PhD $^{6}$, Elizabeth M Brunt, MD $^{7}$, Ann $O$ \\ Scheimann, $\mathrm{MD}^{8}$, Aynur Unalp-Arida, MD, $\mathrm{PhD}^{3}$, and for the NASH Clinical Research \\ Network \\ ${ }^{1}$ Pediatric Gastroenterology, Hepatology and Nutrition, Indiana University School of Medicine \\ ${ }^{2}$ Division of Gastroenterology, Hepatology, and Nutrition, Department of Pediatrics, University of \\ California, San Diego School of Medicine; Department of Gastroenterology, Rady Children's \\ Hospital San Diego; Liver Imaging Group, Department of Radiology, University of California, San \\ Diego School of Medicine \\ ${ }^{3}$ Data Coordinating Center, Johns Hopkins University \\ ${ }^{4}$ Pediatric Gastroenterology, Hepatology and Nutrition, Seattle Children's Hospital \\ ${ }^{5}$ Department of Pathology and Laboratory Medicine, Pediatrics, Indiana University School of \\ Medicine \\ ${ }^{6}$ Pediatric Gastroenterology, Hepatology and Nutrition, Columbia University \\ ${ }^{7}$ Department of Pathology and Immunology, Washington University School of Medicine \\ ${ }^{8}$ Pediatric Gastroenterology and Nutrition, Johns Hopkins Children's Center
}

\section{Abstract}

Objectives-To investigate the histological spectrum of nonalcoholic fatty liver disease

(NAFLD) in children with normal, mildly elevated (26-50 U/L boys, 23-44 U/L girls), or elevated (> 50 boys, $>44$ girls) serum alanine aminotransferase (ALT) levels.

Study design-The Nonalcoholic Steatohepatitis Clinical Research Network (NASH CRN) enrolls children 5-18 years with NAFLD. We analyzed baseline clinical and histological data from 91 children with suspected NAFLD and normal or mildly elevated ALT and liver biopsy within 180 days of ALT, and compared them with 392 children with elevated ALT.

Results-Of 91 children, 17 (19\%) had normal and 74 (81\%) had mildly elevated ALT levels. Overall, $45 \%$ of biopsies had $\geq 33 \%$ steatosis, lobular inflammation grade was $\geq 2$ in $22 \%, 81 \%$ had portal inflammation, $29 \%$ had ballooned hepatocytes, $35 \%$ had "suspicious/borderline"

(C) 2013 Mosby, Inc. All rights reserved.

Corresponding Author: Jean P. Molleston, M.D., Pediatric Gastroenterology, Hepatology and Nutrition, 705 Riley Hospital Drive ROC 4210, Indianapolis, Indiana 46202, Phone: (317) 944-3774, Fax: (317) 944-8521, jpmolles@iu.edu.

* List of members of the NASH CRN is available at www.jpeds.com (Appendix)

Publisher's Disclaimer: This is a PDF file of an unedited manuscript that has been accepted for publication. As a service to our customers we are providing this early version of the manuscript. The manuscript will undergo copyediting, typesetting, and review of the resulting proof before it is published in its final citable form. Please note that during the production process errors may be discovered which could affect the content, and all legal disclaimers that apply to the journal pertain.

The authors declare no conflicts of interest. 
steatohepatitis, and $8 \%$ had definite NASH, 34\% had NAFLD activity score (NAS) $\geq 4$. Overall, $46 \%$ had fibrosis (38\% mild/moderate and $8 \%$ bridging/cirrhosis). Marked steatosis (50\% vs $24 \%$ ) and fibrosis (54\% vs $12 \%$ ) were significantly more common in mildly elevated vs normal, with no difference in ballooning, inflammation, or NAS $\geq 4$. Fibrosis stage $3 / 4$ was seen in none of the children with normal ALT, and in $9 \%$ of the mildly elevated and $15 \%$ of the elevated.

Conclusions-Liver biopsies of children with NAFLD with normal or mildly elevated ALT levels show significant histologic abnormalities, including advanced fibrosis in children with mildly elevated ALT. ALT thus may underestimate liver injury in NAFLD. Appropriate ALT cutoff levels can help identify children at risk for more severe disease.

\section{Keywords}

Nonalcoholic fatty liver disease; alanine aminotransferase; liver histology; children; obesity

Nonalcoholic fatty liver disease (NAFLD) is one of the most common co-morbidities of pediatric obesity. Nonalcoholic steatohepatitis (NASH) can result in cirrhosis, even in childhood. ${ }^{1,2,3}$ The American Academy of Pediatrics Guidelines recommend biannual use of serum alanine aminotransferase (ALT) to screen overweight and obese children for NAFLD. ${ }^{4}$ Elevations in liver enzymes in these children are common. ${ }^{5}$ Data are limited concerning appropriate aminotransferase cut-offs to identify obese children with potential for NASH.

Upper limit of normal (ULN) ALT values used in US children's hospitals vary widely and lack sensitivity to detect NAFLD in children. The median ULN of ALT nationally is 53 U/L and ranges from 30-90 U/L. ${ }^{6}$ Recently, new evidence-based standards for normal ALT in children were proposed-- $\leq 25$ U/L for boys and $\leq 22$ U/L for girls--when NHANES III data were analyzed including only children who had no risk factors for underlying liver disease. ${ }^{6}$.

The NIDDK NASH Clinical Research Network (CRN) maintains a large prospective database of clinical information on children with fatty liver. ${ }^{7}$ We evaluated clinical and histologic variables in children with normal ALT or mildly elevated ALT levels to determine the spectrum of histologic abnormalities and assess the usefulness of ALT in identifying active liver disease. A comparison group of children with elevated ALT levels was examined.

\section{Methods}

The pediatric NAFLD studies were designed by subcommittees of the NASH CRN Steering Committee, the latter composed of principal investigators from each clinical site, the two cochairs of the Pathology Committee, the principal investigator from the Data Coordinating Center (DCC), and the NIDDK scientific officer [all listed in the appendix]. After approval by the Steering Committee, studies were approved by the Institutional Review Boards at each site. Enrolled patients and their guardians gave written informed assent and consent. The protocols, assent/consent forms, and manual of operations were approved by a Data and Safety Monitoring Board established by the NIDDK specifically for the NASH CRN. All studies were in compliance with Good Clinical Practice Guidelines for Human Research Quality Standards.

Children 5 through 18 years of age with definite or suspected NAFLD meeting eligibility criteria, were enrolled into the NIDDK NASH CRN Database or Database 2, which are observational studies conducted at 12 U.S. medical centers ${ }^{8}$ For the initial database study, inclusion criteria defined NAFLD as suggestive radiographic study or local liver biopsy evidence of NAFLD. After 2010 (Pediatric NAFLD Database 2 study), only children who 
had been previously enrolled or had undergone standard-of-care liver biopsy within the 3 months prior to enrollment were eligible. Children were excluded from NASH CRN studies if they had history of alcohol consumption; evidence of other forms of chronic liver disease; history of medicines known to cause fatty liver; history of total parenteral nutrition; biliopancreatic diversion or bariatric surgery; short bowel syndrome; suspected or confirmed hepatocellular carcinoma; known HIV positivity; conditions that were likely to interfere with study follow-up; or inability to provide informed consent. Comprehensive data, including demographics, anthropometrics, medical history, and routine laboratory studies were collected on all patients at entry.

Data included in this analysis were from children enrolled between 2004 and January 2013 in either phase of the NAFLD Database who had histology data from a centrally reviewed biopsy obtained within 6 months of baseline ALT measurement, and whose ALT value close to biopsy was normal ( $\leq 25 \mathrm{U} / \mathrm{L}$ for boys and $\leq 22 \mathrm{U} / \mathrm{L}$ for girls) or mildly elevated (26-50 $\mathrm{U} / \mathrm{L}$ or less for boys and 23-44 U/L or less for girls). A comparison group consisted of children with elevated ALT (> $50 \mathrm{U} / \mathrm{L}$ for boys or $>44 \mathrm{U} / \mathrm{L}$ for girls) and who had had a liver biopsy..

\section{Sample Analyses}

Routine laboratory studies were performed on fresh samples in CLIA certified laboratories at each clinical site according to standard clinical protocols. Liver biopsy specimens were formalin fixed, paraffin embedded and unstained slides were sent to the DCC for inclusion in a central pathology repository. Hematoxylin and eosin (HE), Masson's trichrome and Perls' iron stains were prepared and reviewed centrally by the NASH CRN Pathology Committee, a group of 9 hepatopathologists who were masked to clinical data. Biopsies were scored by consensus during Pathology Committee Meetings using the NAFLD Activity Score (NAS) and fibrosis stage. ${ }^{9}$ The NASH CRN Pathology Committee designed and validated a histological feature scoring system that addresses the full spectrum of lesions of NAFLD for use in clinical trials. The scoring system comprised 14 histological features, 4 of which were evaluated semiquantitatively: steatosis (0-3), lobular inflammation (0-3), hepatocellular ballooning (0-2), fibrosis (0-4) and other features were recorded as present or absent. Diagnoses are also rendered: definite steatohepatitis, borderline $1 \mathrm{a}$ or $1 \mathrm{~b}$ (centrilobular accentuation or portal accentuation, respectively) and definitely not steatohepatitis. The NAS was determined by summing the unweighted scores for steatosis, lobular inflammation and hepatocellular ballooning (0-8).

\section{Data Analyses}

Cross-sectional analyses of demographic factors including sex, age, race (white vs nonwhite) and ethnicity; anthropometrics including Tanner stage (stage 1 vs other), body mass index (BMI) z score; and the history of co-morbid conditions including diabetes, hyperlipidemia, and hypertension (reported diagnosis or on current treatment) were performed. We also examined serum levels of aspartate aminotransferase (AST), gamma glutamyl transpeptidase (GGT), triglycerides, total cholesterol, HDL and LDL cholesterol, hemoglobin A1c (HbA1c), fasting glucose and insulin as well as the homeostasis model assessment (HOMA) index. Histological features analyzed included the fibrosis stage (none, mild/moderate (1-2), bridging/cirrhosis (3-4), NAS, presence of definite or borderline $\mathrm{NASH}$, steatosis grade, lobular inflammation $(<2, \geq 2)$, portal inflammation (none, mild or more), hepatocellular ballooning (none, few to many), acidophil bodies (rare/absent, many).

Data are reported as means and standard deviations or percents. The significance of differences between the groups were assessed using either Fisher exact test for $2 \times 2$ categorical variables, Cochran-Armitage exact trend test for $2 \times 3$ categorical variables, or 
Kruskal-Wallis test for continuous variables. ${ }^{10}$ All analyses were conducted using SAS $9.3^{11}$ and Stata (version 12) softwares. ${ }^{12}$ Nominal, two-sided $\mathrm{P}$ values were presented.

\section{Results}

Of 483 children ages 5-18 enrolled in the NASH CRN database study from October 2004 until January 2013, 91 children had normal or mildly elevated ALT with a centrally reviewed liver biopsy within 180 days of baseline ALT measure; 32 (35\%) were enrolled in NAFLD Database and $59(65 \%)$ in NAFLD Pediatric Database 2 Study. Among the elevated comparison group consisting of 392 children, $11 \%$ were enrolled in NAFLD Database, $46 \%$ in NAFLD Pediatric Database 2, and $43 \%$ in a treatment trial.

The reasons for NAFLD evaluation reported by parent at entry interview in the normal or mildly elevated subjects included: symptoms of liver disease (22\%), results of evaluation for other illness (38\%), during routine physical exam (41\%), blood donation (2\%), other (4\%). By history, data that supported the first diagnosis of NAFLD in children included: elevated ALT (74\%), liver biopsy (53\%), and radiographic evidence of steatosis (55\%). Children were predominantly male, Caucasian, and Hispanic. Mean age of the children with normal or mildly elevated ALT was $12.9 \pm 2.8$ years and three-quarters were pubescent; $69 \%$ were moderately to severely obese, with BMI Z score $\geq 2$. Mean HOMA-IR was $6.3 \pm 4.5$, with relatively normal mean cholesterol and triglycerides; $10 \%$ of the children with normal or mildly elevated ALT had been diagnosed with diabetes, $8 \%$ with hyperlipidemia, and $7 \%$ percent with hypertension (Table I; available at www.jpeds.com).

Liver biopsies were obtained within an average of 58 days (range 5 to 154) of blood collection for ALT determination at enrollment for the normal group and 47 days (0-175) of enrollment ALT for the mildly elevated group. Regression analysis (not shown) showed no interaction effects between interval from ALT to biopsy and ALT group. In these children with normal or mildly elevated ALT, steatosis was more than grade 2 (> 33\%) in $45 \%$ (Table I). Lobular inflammation grade was 2 or more in $22 \%$, and $81 \%$ had some degree of portal inflammation. Ballooning was seen in $29 \%$ and many acidophil bodies were present in $29 \%$. Fibrosis was present in almost half of the biopsies and was distributed as stage 1 or 2 fibrosis (38\%) and bridging fibrosis or cirrhosis (8\%). The diagnosis of steatohepatitis was borderline in $35 \%$ and definite in $8 \%$ (distribution among ALT groups shown in Figure 1). A NAS of 4 or more was seen in 34\% (Table I).

Subgroup analysis comparing children with normal ALT and those with mildly elevated ALT is shown in Table I. Children with mildly elevated ALT were younger than those with normal ALT but had higher BMI Z-score. Both AST and GGT were significantly lower in the normal group. HOMA-IR did not differ between these groups. Steatosis grade was significantly lower in the normal ALT group: greater than 33\% steatosis was seen in $24 \%$ of the normal group compared with $50 \%$ of the mildly elevated group $(\mathrm{p}=0.02)$. Fibrosis, seen in $54 \%$ of the mildly elevated group, was significantly $(\mathrm{p}<0.003)$ more frequent than in normal group (12\%) (Figure 2). In the normal group, two biopsies showed periportal fibrosis (stage 1c), but there was no child with advanced fibrosis. Notably, there were no significant differences between groups in the rates or distribution of inflammation, ballooning, steatohepatitis diagnosis, or NAFLD activity score < 4 vs. $\geq 4$. However, the mildly elevated group compared with the normal ALT group contained fewer children with "no NASH" and all of the children with the diagnostic category of "definite" NASH ( $\mathrm{p}=0.61)$

Comparison of the mildly elevated vs elevated ALT groups is shown in Table II. Mean ALT for elevated was 123.1 and for mildly elevated was 37.4. Significant findings included higher AST (mean 71.9 vs 31.6), GGT (mean 50.7 vs 29.8), percent with moderate/severe 
steatosis ( $74 \%$ vs $50 \%$ ), lobular inflammation $\geq$ grade 2 ( $48 \%$ vs $20 \%$ ), ballooning ( $48 \%$ vs $31 \%$ ), bridging fibrosis/cirrhosis ( $15 \%$ vs $9 \%$ ), definite NASH (30\% vs $9 \%$ ), and high NAS ( $72 \%$ vs $36 \%$ ) in those with elevated liver enzymes compared with those with mildly elevated liver enzymes (Figure 2).

\section{Advanced Fibrosis}

The 7 children with advanced fibrosis (stages 3 or 4 ) and mildly elevated ALT were all male, younger (average age $12 \pm 3$ years), mostly Hispanic ( 5 of 7 ), obese (average BMI Z score $2.46 \pm 0.55$ ), and insulin-resistant (HOMA $5.32 \pm 3.09$ ). None had ever been diagnosed with diabetes, hypertension, or hyperlipidemia. Only one of these children had fluctuating ALT subsequent to the study value (data not shown). Most biopsies with advanced fibrosis did not have a high NAS score: 6 of the 7 had a NAS score of 4 or less. Ballooning was only seen in $29 \%$ but all had at least mild portal inflammation. Four were diagnosed as borderline steatohepatitis and 3 were diagnosed as "not steatohepatitis". Fibrosis stage was 3 in all but one, who had cirrhosis, stage 4 (Table III; available at www.jpeds.com).

\section{Discussion}

NAFLD is the most common chronic liver disease in pediatrics, occurring in $9.6 \%$ of children and as many as $38 \%$ of obese children. ${ }^{3,13}$ Liver disease can range from isolated hepatic steatosis to steatohepatitis with active inflammation to cirrhosis. ${ }^{9}$.. Ultrasound is often used as a noninvasive means to screen for steatosis; it has only a moderate positive predictive value, does not detect all cases, and reflects fat and sometimes fibrosis, but not inflammation or injury. ${ }^{14}$ Serum ALT levels, a surrogate for necroinflammatory activity, is sometimes used as a proxy for disease activity. Biannual screening of obese children for ALT has been recommended by an expert panel with representatives from 15 different societies including the American Academy of Pediatrics, overseen by the Center for Disease Control (CDC) and Maternal and Child Health Branch of HRSA. Evidence as to the utility and cost-effectiveness of this approach is still lacking and such a recommendation to monitor ALT in obese children was not made in the recent American Association for the Study of Liver Diseases NAFLD Management Guidelines. ${ }^{15}$ Furthermore, appropriate ALT criteria for referral (the expert committee recommended "twice ULN"), biopsy, or more aggressive interventions have not been established. Using ultrasound as a standard, the sensitivity of using ALT $>30$ or $40 \mathrm{U} / \mathrm{L}$ as a cut-off for identifying NAFLD is poor. ${ }^{16}$. Liver biopsy is the clinical standard for semi-quantitatively evaluating fat, hepatocellular injury, and evaluation of fibrosis. However, the decision of whether or not to perform a liver biopsy is challenging and includes the consideration of cost and risk. ${ }^{17}$

The average ULN ALT in pediatric hospitals in the United States is $53 \mathrm{U} / \mathrm{L} .{ }^{6}$ A recent report demonstrated, by excluding children with obesity, viral hepatitis, history of potentially hepatotoxic medications, and other potential causes of liver injury, that the $95^{\text {th }}$ percentile ULN ALT for girls is $22 \mathrm{U} / \mathrm{L}$ and for boys is $25 \mathrm{U} / \mathrm{L}$. Using a cut-off of $50 \mathrm{U} / \mathrm{L}$ for boys and $44 \mathrm{U} / \mathrm{L}$ for girls, twice the suggested ULN, we analyzed the degrees of steatosis, inflammation, hepatocyte injury, and fibrosis in 91 children with prospectively assessed data and blinded pathology review from the NASH CRN. We demonstrated the presence of considerable inflammation, ballooning, acidophil bodies and fibrosis in children with NAFLD and stringently defined normal or mildly elevated ALT.

Biopsies in children with normal ALT were noted to have definite histologic abnormalities, but not important fibrosis. Even among children with normal ALT (half of whom had BMI $\mathrm{Z}$ score $<2$ ), $24 \%$ had NAS scores in the 4-8 range and almost $30 \%$ had borderline NASH; the degree of steatosis in these children was less than those with higher ALT, however, and 
only 2 of 17 biopsies had any fibrosis (mild to moderate). Biopsies in those subjects with mildly elevated ALT demonstrated significantly more steatosis and fibrosis than the normal group and included 7 children with advanced fibrosis (stages 3-4). Inflammation, ballooning, or rate of NAS $>4$ were not greater in this mildly elevated group, which contained children who were younger but heavier than those in the normal group. Most of the children with mildly elevated ALT and advanced fibrosis did not have NAS $>4$. They thus did not fulfill the typical criteria for NASH, reinforcing the idea that pediatric NASH is a heterogeneous disorder and that histologic activity of NASH may decrease as fibrosis and architectural remodeling increase. Even more notable histologic abnormalities and significantly more fibrosis were seen in the elevated ALT comparison group. Overall, histological abnormalities occurred in all ALT groups, including those previously considered by many as normal.

In a retrospective single-center report on 106 children with NAFLD, A-Kader et al reported fibrosis in 10 of 15 children with normal ALT ( $31 \pm 1.6 \mathrm{IU} / \mathrm{L}) ; 2$ children had stage 2 (central and portal) or 3 (bridging) fibrosis. ${ }^{18}$ Manco et al showed no difference in mean ALT levels in children with NAS $<2,3-4$, or $>5 .{ }^{19}$ They reported steatosis grade 2-3, necroinflammatory grade $2-3$, and fibrosis stage $2-4$ in $68.1 \%, 4.7 \%$, and $81.4 \%$, respectively (fibrosis stage was 3 or 4 in 11.6\%) in a sample of 43 children with "normal ALT" (not defined) ${ }^{20}$ In a previous report from the NASH CRN in a prospectively studied group of 176 children (with mean ALT of 90), ALT increased with higher NAS: mean ALT was 74 with NAS $1-3,87$ with NAS 4-5, and 114 with NAS 6-7 (P-trend <0.001). The area under the ROC curve for ALT comparing none/mild fibrosis vs moderate fibrosis was $0.62 .^{2}$.

Biopsy data in adults with low ALT are available. Fracanzani reported results of 4 adult NASH databases in Italy, finding NASH in $59 \%$ of adult patients with ALT $<40 \mathrm{U} / \mathrm{L}^{21}$ Mofrad et al described the clinical and histologic spectrum of NAFLD;. of 51 adults with normal ALT (defined as ALT $<75 \mathrm{U} / \mathrm{L}$ ), 12 had bridging fibrosis and 6 had cirrhosis 22 . Although steatosis and fibrosis were less in 15 subjects whose ALT was below $30 \mathrm{U} / \mathrm{L}$, the prevalence of advanced fibrosis was similar in both groups.

The strengths of our study include prospective evaluation of a large and well-characterized multicenter population of children with NAFLD and low or mildly elevated ALT. Biopsies were assessed by pathologists blinded to age and all clinical information at the time of review using a structured and validated scoring system. Several weaknesses are inherent. We studied a selected population of children who underwent biopsy for evaluation of NAFLD; indications for this standard-of-care biopsy varied, making generalizability of these data to the general population unclear. Furthermore, we evaluated only one ALT measurement, the one closest to biopsy; some subjects had a prior history of elevated ALT leading to initial evaluation for NAFLD. Although on average biopsy was done within a clinically realistic 47-58 days of ALT determination, biopsies up to 176 days from ALT were included. Our regression analysis, however, showed no significant interactions between timing of biopsy and ALT group. It is conceivable that the disease process could have evolved during that time interval, perhaps associated with weight changes. We cannot exclude previous or subsequent fluctuations of ALT in these subjects. Factors such as BMI, recent diet, time of day, race, ethnicity, and storage conditions can influence ALT. ${ }^{23}$ Few of the children enrolled in the NASH CRN Database studies had ALT values < $50 \mathrm{U} / \mathrm{L}$ for boys and $<44 \mathrm{U} / \mathrm{L}$ for girls; therefore, the numbers available for multivariable analyses comparing the 2 groups and controlling for some of these factors were inadequate.

NASH is a serious cause of liver disease in children. The fact that diabetes (10\%), hyperlipidemia (8\%), and hypertension (7\%) are so common in children with normal or 
mildly elevated ALT in this study underscores the metabolic impact of obesity in this group. This study shows that important histologic abnormalities can occur in this group, and implies that they might progress, unheralded by liver enzyme abnormalities, to more advanced liver disease over time. Children with fatty liver who have mildly elevated ALT may have that level reported as normal by many labs, yet may have significant liver disease. Even when stringently defined, normal ALT does not signify lack of liver injury in children with fatty liver; clinicians should be aware that serious liver disease may be present in these children. Liver biopsy has a key role in the evaluation of children with NAFLD as histology remains the gold standard for the assessment of NAFLD. The development of more informative noninvasive biomarkers for NAFLD activity and fibrosis is crucial in children as well as in adults.

\section{Supplementary Material}

Refer to Web version on PubMed Central for supplementary material.

\section{Acknowledgments}

Supported by the National Institute of Diabetes and Digestive and Kidney Diseases (U01DK061718, U01DK061728, U01DK061731, U01DK061732, U01DK061734, U01DK061737, U01DK061738, U01DK061730, U01DK061713), and the National Institute of Child Health and Human Development Several clinical centers use support from General Clinical Research Centers or Clinical and Translational Science Awards in conduct of NASH CRN Studies (UL1RR024989, UL1RR025761, M01RR00188, UL1RR024131, UL1RR025014, UL1RR031990, UL1RR025741, UL1RR029887, UL1RR24156, UL1RR025055, and UL1RR031980).

\section{List of abbreviations and acronyms that appear $>3$ times}

NAFLD Nonalcoholic fatty liver disease

ALT

NASH

NASH CRN

U/L

ULN

DCC

NAS

BMI

AST

GGT

HbA1c

HOMA-IR
Alanine aminotransferase

Nonalcoholic steatohepatitis

Nonalcoholic Steatohepatitis Clinical Research Network

Units per liter

Upper limit of normal

Data Coordinating Center

NAFLD Activity Score

Body mass index

Aspartate aminotransferase

Gamma glutamyl transpeptidase

Hemoglobin A1c

Homeostasis model assessment

\section{References}

1. Alisi A, Manco M, Vania A, Nobili V. Pediatric Nonalcoholic Fatty Liver Disease in 2009. J Pediatr. 2009; 155:469-474. [PubMed: 19772998]

2. Patton HM, Lavine JE, Van Natta ML, Schwimmer JB, Kleiner D, Molleston J. Clinical correlates of histopathology in pediatric nonalcoholic steatohepatitis. Gastroenterology. 2008; 135:1961-1971. [PubMed: 19013463] 
3. Lavine JE, Schwimmer JB. Nonalcoholic fatty liver disease in the pediatric population. Clin Liver Dis. 2004; 8:549-558. [PubMed: 15331063]

4. Barlow SE. Expert committee recommendations regarding the prevention, assessment, and treatment of child and adolescent overweight and obesity: summary report. Pediatrics. 2007; 120:S164-S192. [PubMed: 18055651]

5. Strauss RS, Barlow SE, Dietz WH. Prevelance of abnormal serum aminotransferase values in overweight and obese adolescents. J Pediatr. 2000; 136:727-733. [PubMed: 10839867]

6. Schwimmer JB, Dunn W, Norman GJ, Pardee PE, Middleton MS, Kerkar N, et al. SAFETY study: alanine aminotransferase cutoff values are set too high for reliable detection of pediatric chronic liver disease. Gastroenterology. 2010; 138:1357-1364. [PubMed: 20064512]

7. Lavine JE, Schwimmer JB, Molleston JP, Scheimann AO, Murray KF, Abrams SH, et al. Treatment of nonalcoholic fatty liver disease in children: TONIC trial design. Contemp Clin Trials. 2010; 31:62-70. [PubMed: 19761871]

8. Neuschwander-Tetri BA, Clark JM, Bass NM, Van Natta ML, Unalp-Arida A, Tonascia J, et al. Clinical, laboratory and histological associations in adults with nonalcoholic fatty liver disease. Hepatology. 2010; 52:913-924. [PubMed: 20648476]

9. Kleiner DE, Brunt EM, Van Natta M, Behling C, Contos MJ, Cummings OW, et al. Design and validation of a histological scoring system for nonalcoholic fatty liver disease. Hepatology. 2005; 41:1313-1321. [PubMed: 15915461]

10. Agresti, A. Categorical Data Analysis. 1st ed.. New York: Wiley, John \& Sons, Inc.; 1990.

11. SAS Institute I. SAS software, version 9.3 of the SAS system for windows. Cary, NC: 2002-2010.

12. StataCorp. Stata statistical software: release 12. College Station, TX: StataCorp LP; 2011.

13. Schwimmer JB, Deutsch R, Kahen T, Lavine JE, Stanley C, Behling C. Prevalence of fatty liver in children and adolescents. Pediatrics. 2006; 118:1388-1393. [PubMed: 17015527]

14. Devadason CA, Scheimann AO. Overview of screening methods for fatty liver disease in children. World J Hepatol. 2012; 4:1-4. [PubMed: 22312449]

15. Chalasani N, Younossi Z, Lavine JE, Diehl AM, Brunt EM, Cusi K, et al. The diagnosis and management of non-alcoholic fatty liver disease: practice guideline by the American Association for the Study of Liver Diseases, American College of Gastroenterology, and the American Gastroenterological Association. Hepatology. 2012; 55:2005-2023. [PubMed: 22488764]

16. Sartorio A, Del Col A, Agosti F, Mazzilli G, Bellentani S, Tiribelli C, et al. Predictors of nonalcoholic fatty liver disease in obese children. Eur J Clin Nutr. 2006; 61:877-883. [PubMed: 17151586]

17. Ovchinsky N, Moreira RK, Lefkowitch JH, Lavine JE. Liver Biopsy in Modern Clinical Practice: A Pediatric Point-of-View. Adv Anat Pathol. 2012; 19:250-262. [PubMed: 22692288]

18. A-Kader HH, Henderson J, Vanhoesen K, Ghishan F, Bhattacharyya A. Nonalcoholic Fatty Liver Disease in Children: A Single Center Experience. Clinical Gastroenterology and Hepatology. 2008; 6:799-802. [PubMed: 18486560]

19. Manco M, Marcellini M, DeVito R, Comparcola D, Sartorelli MR, Nobili V. Metabolic syndrome and liver histology in paediatric non-alcoholic steatohepatitis. Int J Obes. 2007; 32:381-387.

20. Manco M, Alisi A, Nobili V. Risk of severe liver disease in NAFLD with normal ALT levels: A pediatric report. Hepatology. 2008; 48:2087-2088. [PubMed: 18980229]

21. Fracanzani AL, Valenti L, Bugianesi E, Andreoletti M, Colli A, Vanni E, et al. Risk of severe liver disease in nonalcoholic fatty liver disease with normal aminotransferase levels: a role for insulin resistance and diabetes. Hepatology. 2008; 48:792-798. [PubMed: 18752331]

22. Mofrad P, Contos MJ, Haque M, Sargeant C, Fisher RA, Luketic VA, et al. Clinical and histologic spectrum of nonalcoholic fatty liver disease associated with normal ALT values. Hepatology. 2003; 37:1286-1292. [PubMed: 12774006]

23. Dufour DR, Lott JA, Nolte FS, Gretch DR, Koff RS, Seeff LB. Diagnosis and Monitoring of Hepatic Injury. I. Performance Characteristics of Laboratory Tests. Clin Chem. 2000; 46:20272049. [PubMed: 11106349] 


\section{Appendix}

Members of Nonalcoholic Steatohepatitis Clinical Research Network Pediatric Clinical Centers include:

Baylor College of Medicine, Houston, TX: Stephanie H. Abrams, MD, MS; Leanel Angeli Fairly, RN; Case Western Reserve University Clinical Centers: MetroHealth Medical Center, Cleveland, OH: Carol Hawkins, RN; Margaret Stager, MD (2004-2009); Children's National Medical Center, Washington DC (2007-2009): Parvathi Mohan, MD (2007-2009); Cincinnati Children's Hospital Medical Center, Cincinnati, OH: Stephanie DeVore, MSPH; Rohit Kohli, MD; Kathleen Lake, MSW; Stavra Xanthakos, MD; Columbia University, New York, NY: Joel E. Lavine, MD, PhD; Ali Mencin, MD; Nadia Ovchinsky, MD; Indiana University School of Medicine, Indianapolis, IN: Elizabeth Byam, RN; Oscar W. Cummings, MD; Ann Klipsch, RN; Jean P. Molleston, MD; Girish Subbarao, MD; Johns Hopkins Hospital, Baltimore, MD: Caroline Devadason; Kimberly Pfeifer, RN; Ann Scheimann, MD; Michael Torbenson, MD; Mount Sinai Kravis Children's Hospital, New York, NY: Nanda Kerkar, MD (2010-2012); Frederick Suchy, MD (2010); Northwestern University Feinberg School of Medicine/Children's Memorial Hospital: Katherine Dunne; Mark H. Fishbein, MD; Katie Jacques; Ann Quinn, RD; Cindy Riazi, RN; Peter F. Whitington, MD; Saint Louis University, St Louis, MO: Sarah Barlow, MD (2002-2007); Jose Derdoy, MD; Debra King, RN; Andrea Morris; Joan Siegner, RN; Susan Stewart, RN; Brent A. Neuschwander-Tetri, MD; Judy Thompson, RN; University of California San Diego, San Diego, CA: Cynthia Behling, MD, PhD; Jennifer Collins; Janis Durelle; Anya Morgan; Steven Rose, MD (2007-2009); Jeffrey B. Schwimmer, MD; Claude Sirlin, MD; Tanya Stein, MD (2005-2009); University of California San Francisco, San Francisco, CA: Linda D. Ferrell, MD; Danuta Filipowski, MD (2005-2010); Shannon Fleck; Camille Langlois; Philip Rosenthal, MD; University of Washington Medical Center and Seattle Children's Hospital, Seattle, WA: Melissa Young; Deana Rich; Karen Murray, MD; Matthew Yeh, MD, PhD; Virginia Commonwealth University, Richmond, VA: Sherry Boyett, RN, BSN; Melissa J. Contos, MD; Michael Fuchs, MD; Amy Jones; Velimir AC Luketic, MD; Puneet Puri, MD; Bimalijit Sandhu, MD (2007-2009); Arun J. Sanyal, MD; Carol Sargeant, RN, BSN, MPH; Kimberly Noble; Melanie White, RN, BSN (2006-2009); Washington University School of Medicine, St. Louis, MO: Elizabeth M. Brunt, MD; Resource Centers; National Cancer Institute, Bethesda, MD: David E. Kleiner, MD, PhD; National Institute of Child Health and Human Development, Bethesda, MD: Gilman D. Grave, MD; National Institute of Diabetes and Digestive and Kidney Diseases, Bethesda, MD: Edward C. Doo, MD; Jay H. Hoofnagle, MD; Patricia R. Robuck, PhD, MPH; Averell Sherker, MD; Johns Hopkins University, Bloomberg School of Public Health (Data Coordinating Center), Baltimore, MD: Patricia Belt, BS; Jeanne M. Clark, MD, MPH; Michele Donithan, MHS; Milana Isaacson, BS; Kevin P. May, MS; Laura Miriel, BS; Alice Sternberg, ScM; James Tonascia, PhD; Aynur Ünalp-Arida, MD, PhD; Mark Van Natta, MHS; Ivana Vaughn, MPH; Laura Wilson, ScM; Katherine Yates, ScM. 


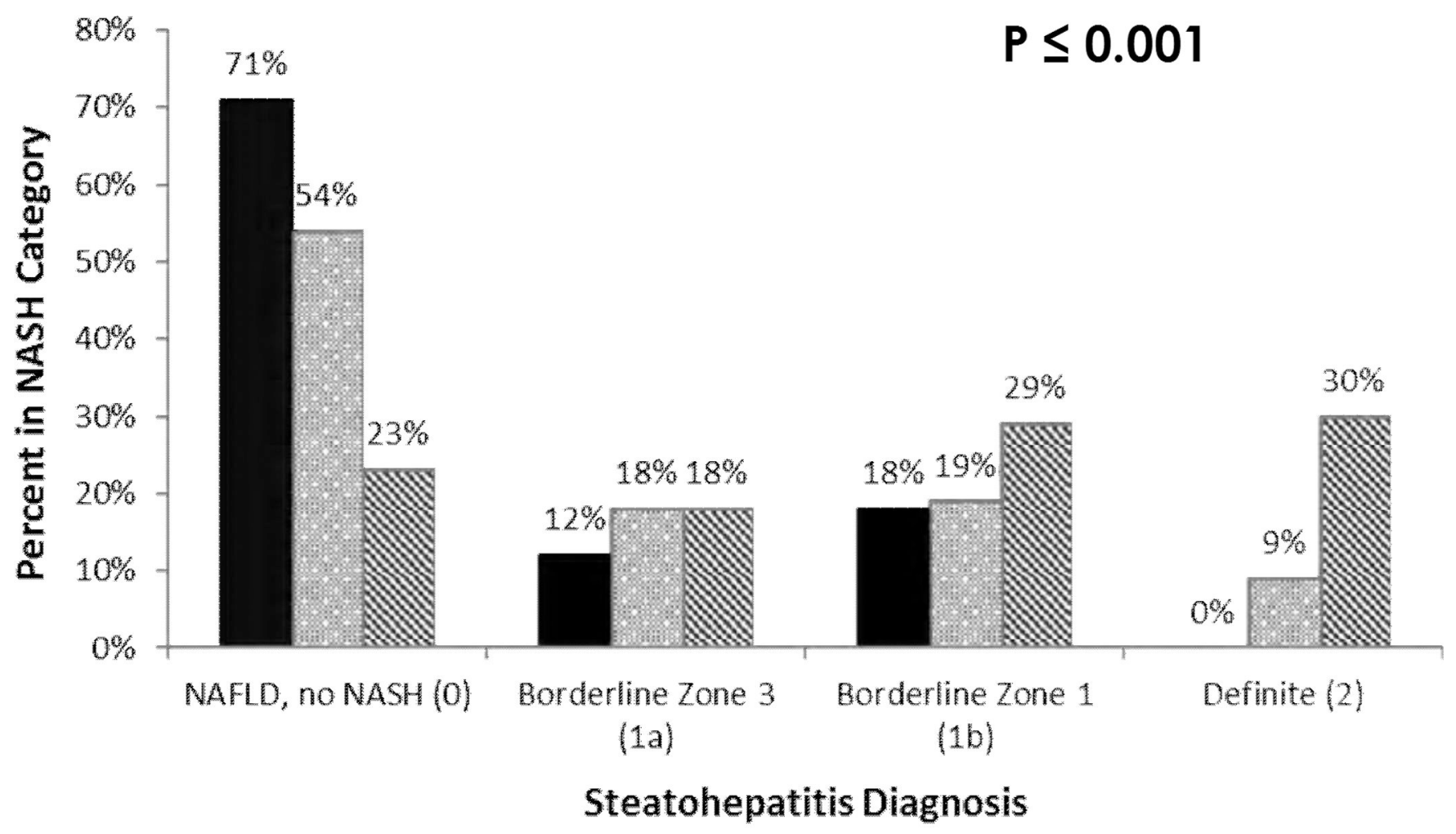

\section{normal Mildy elevated Elevated}

Figure 1.

Percent of children in each NASH diagnosis category for normal, mildly elevated, and elevated ALT. NASH diagnosis categories are NAFLD/no NASH, Borderline Zone 3 (1a), Borderline Zone 1 (1b), Definite (2). 


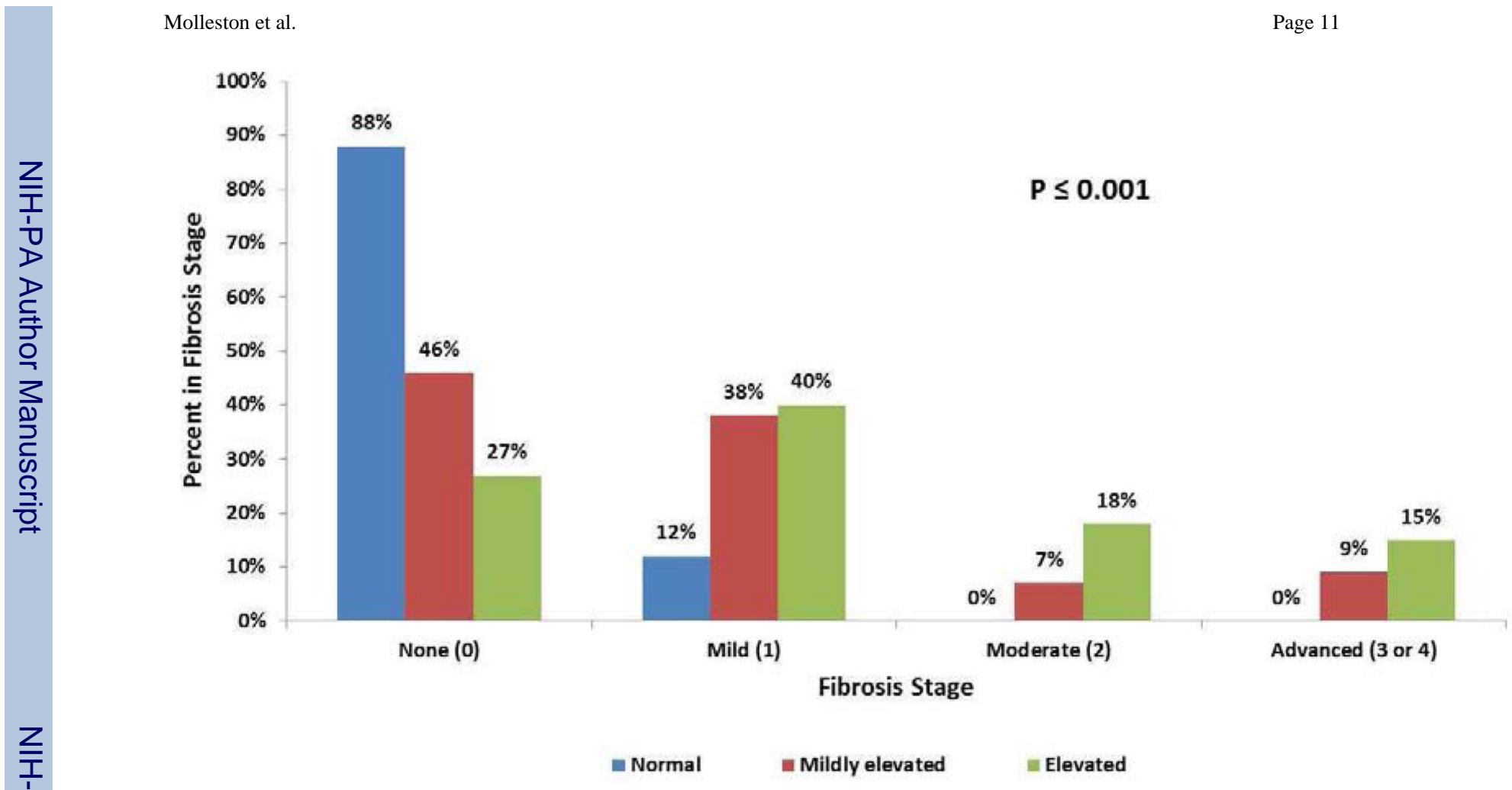

Figure 2.

Percent of biopsies in fibrosis stages None, Mild, Moderate, and Advanced for each ALT group. 


\section{Table I}

Baseline Demographic, Clinical and Histologic Characteristics of Children with NAFLD Comparing Normal and Mildly Elevated Levels of Alanine Aminotransferase (ALT)

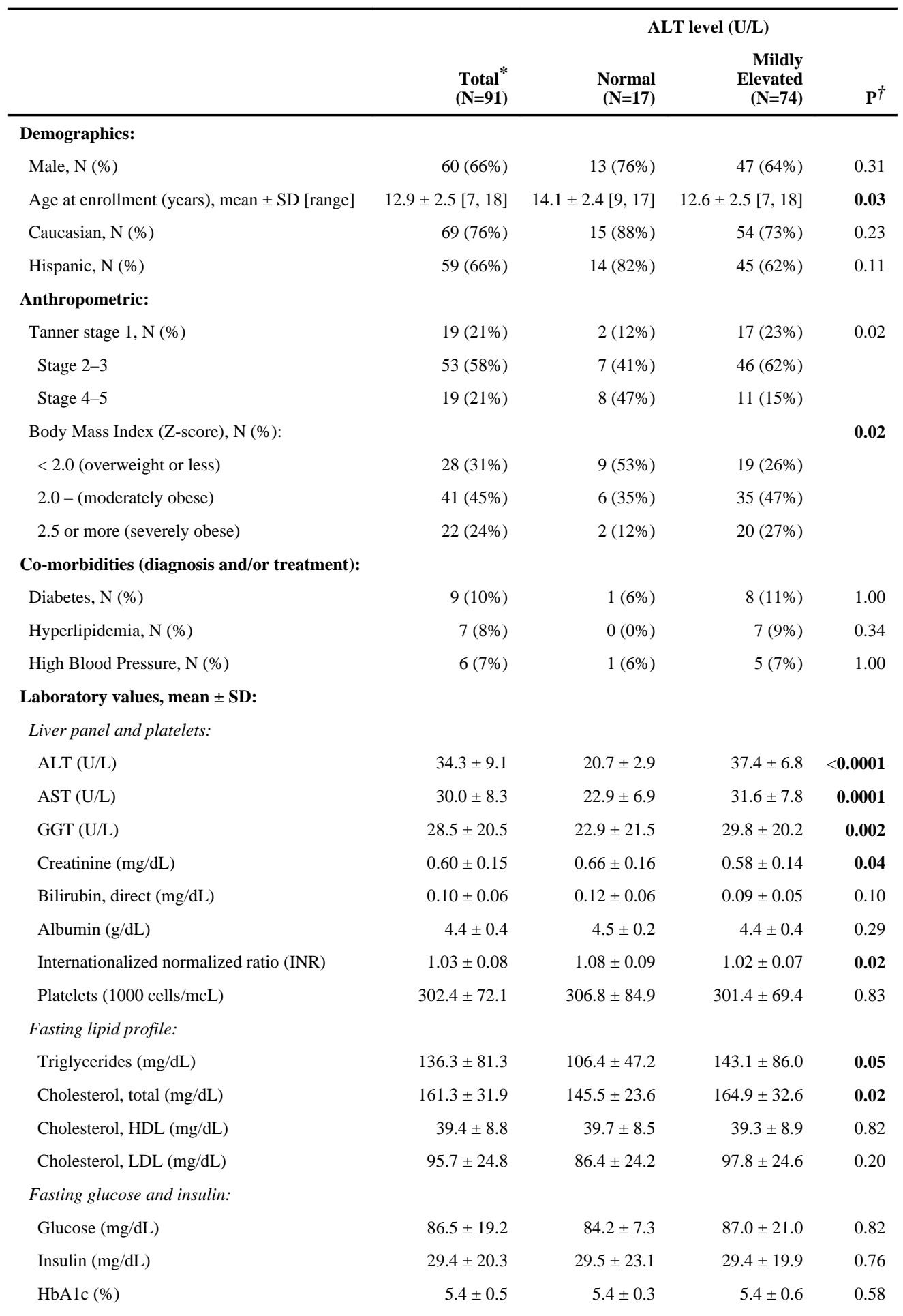




\begin{tabular}{|c|c|c|c|c|}
\hline & \multicolumn{4}{|c|}{ ALT level (U/L) } \\
\hline & $\begin{array}{l}\text { Total }^{*} \\
(\mathrm{~N}=91)\end{array}$ & $\begin{array}{r}\text { Normal } \\
(\mathbf{N}=\mathbf{1 7})\end{array}$ & $\begin{array}{r}\text { Mildly } \\
\text { Elevated } \\
(\mathrm{N}=74)\end{array}$ & $\mathbf{P}^{\dagger}$ \\
\hline HOMA-IR & $6.3 \pm 4.5$ & $6.0 \pm 4.8$ & $6.3 \pm 4.4$ & 0.69 \\
\hline \multicolumn{5}{|l|}{ Histologic characteristics, $N(\%)$ : } \\
\hline Steatosis grade: & & & & 0.02 \\
\hline $0-4 \%$ & $9(10 \%)$ & $4(24 \%)$ & $5(7 \%)$ & \\
\hline $5-33 \%$ (mild) & $41(45 \%)$ & $9(53 \%)$ & $32(43 \%)$ & \\
\hline$>33 \%$ (moderate or severe) & $41(45 \%)$ & $4(24 \%)$ & $37(50 \%)$ & \\
\hline Lobular inflammation, $20 \times$ mag: & & & & 0.52 \\
\hline$<2$ & $71(78 \%)$ & $12(71 \%)$ & $59(80 \%)$ & \\
\hline$\geq 2$ & $20(22 \%)$ & $5(29 \%)$ & $15(20 \%)$ & \\
\hline Portal chronic inflammation: & & & & 0.73 \\
\hline None & $17(19 \%)$ & $4(24 \%)$ & $13(18 \%)$ & \\
\hline Mild/More than mild & $74(81 \%)$ & $13(76 \%)$ & $61(82 \%)$ & \\
\hline Ballooning injury: & & & & 0.38 \\
\hline None & $65(71 \%)$ & $14(82 \%)$ & $51(69 \%)$ & \\
\hline Few/many & $26(29 \%)$ & $3(18 \%)$ & $23(31 \%)$ & \\
\hline Acidophil bodies: & & & & 1.00 \\
\hline Rare/absent & $65(71 \%)$ & $12(71 \%)$ & $53(72 \%)$ & \\
\hline Many & $26(29 \%)$ & $5(29 \%)$ & $21(28 \%)$ & \\
\hline Fibrosis stage: & & & & 0.002 \\
\hline None $(0)$ & $49(54 \%)$ & $15(88 \%)$ & $34(46 \%)$ & \\
\hline \multicolumn{5}{|l|}{ Mild (1) } \\
\hline Perisinusoidal, Zone $3(1 \mathrm{a})^{\wedge}$ & $6(7 \%)$ & $0(0 \%)$ & $6(8 \%)$ & \\
\hline Perisinusoidal, Zone $3,(1 \mathrm{~b})^{\wedge}$ & $1(1 \%)$ & $0(0 \%)$ & $1(1 \%)$ & \\
\hline Portal/periportal (1c) & $23(25 \%)$ & $2(12 \%)$ & $21(28 \%)$ & \\
\hline \multicolumn{5}{|l|}{ Moderate (2) } \\
\hline Perisinusoidal and portal, periportal & $5(5 \%)$ & $0(0 \%)$ & $5(7 \%)$ & \\
\hline \multicolumn{5}{|l|}{ Severe ( 3 or 4$)$} \\
\hline Bridging fibrosis or cirrhosis & $7(8 \%)$ & $0(0 \%)$ & $7(9 \%)$ & \\
\hline Steatohepatitis diagnosis: & & & & 0.61 \\
\hline NAFLD, not NASH & $52(57 \%)$ & $12(71 \%)$ & $40(54 \%)$ & \\
\hline Suspicious/borderline Zone 3 (1a) & $15(16 \%)$ & $2(12 \%)$ & $13(18 \%)$ & \\
\hline Suspicious/borderline Zone 1 (1b) & $17(19 \%)$ & $3(18 \%)$ & $14(19 \%)$ & \\
\hline Definite & $7(8 \%)$ & $0(0 \%)$ & $7(9 \%)$ & \\
\hline NAFLD Activity Score: & & & & 0.31 \\
\hline $0-3$ & $60(66 \%)$ & $13(76 \%)$ & $47(64 \%)$ & \\
\hline $4-8$ & $31(34 \%)$ & $4(24 \%)$ & $27(36 \%)$ & \\
\hline Mean \pm SD & $3.1 \pm 1.2$ & $2.6 \pm 1.3$ & $3.2 \pm 1.2$ & 0.05 \\
\hline
\end{tabular}

* All participants who were ages 5 through 18 at enrollment in with a centrally reviewed biopsy within 180 days of enrollment or blood draw for the ALT measure and diagnosed with NAFLD by either the locally or centrally read biopsy were included in the study.

J Pediatr. Author manuscript; available in PMC 2015 April 01. 
No. missing values for a characteristic reported in parentheses by ALT group $(0-25,26-50)$ : Hispanic $(0,1)$, Cholesterol: LDL $(0,1,6)$, Insulin, HOMA-IR $(2,5)$, INR $(0,1)$

${ }^{\dagger} \mathrm{P}$ values (2-sided) determined from either a Fisher exact test, a chi-square test or a Cochran-Armitage test for trend (for ordered categories) for categorical variables or a Wilcoxon Rank sum test for continuous variables.

$\hat{1 a}$ fibrosis requires trichrome stain, $1 \mathrm{~b}$ fibrosis can be visualized by HE stain. 
Table II

Demographic, Clinical and Histologic Characteristics of Pediatric Participants with NAFLD Comparing Mildly Elevated and Elevated Levels of Alanine Aminotransferase (ALT)

\begin{tabular}{|c|c|c|c|}
\hline & \multicolumn{3}{|c|}{$\operatorname{ALT}$ level (U/L) ${ }^{*}$} \\
\hline & $\begin{array}{r}\text { Mildly } \\
\text { Elevated } \\
(N=74)\end{array}$ & $\begin{array}{c}\text { Elevated } \\
(\mathbf{N}=392)\end{array}$ & $\mathbf{P}^{\dagger}$ \\
\hline \multicolumn{4}{|l|}{ Demographics: } \\
\hline Male, $\mathrm{N}(\%)$ & $47(64 \%)$ & $293(75 \%)$ & 0.05 \\
\hline Age at enrollment (years), mean \pm SD [range] & $12.6 \pm 2.5[7,18]$ & $12.6 \pm 2.7[5,18]$ & 0.84 \\
\hline Caucasian, $\mathrm{N}(\%)$ & $54(73 \%)$ & $304(78 \%)$ & 0.37 \\
\hline Hispanic, $\mathrm{N}(\%)$ & $45(62 \%)$ & $246(63 \%)$ & 0.86 \\
\hline \multicolumn{4}{|l|}{ Anthropometric: } \\
\hline Tanner stage $1, \mathrm{~N}(\%)$ & $17(23 \%)$ & $131(33 \%)$ & 0.37 \\
\hline Stage $2-3$ & $46(62 \%)$ & $192(49 \%)$ & \\
\hline Stage 4-5 & $11(15 \%)$ & $69(18 \%)$ & \\
\hline Body Mass Index (Z-score), $\mathrm{N}(\%)$ : & & & 0.26 \\
\hline$<2.0$ (overweight or less) & $19(26 \%)$ & $82(21 \%)$ & \\
\hline $2.0-$ (moderately obese) & $35(47 \%)$ & $182(46 \%)$ & \\
\hline 2.5 or more (severely obese) & $20(27 \%)$ & $128(33 \%)$ & \\
\hline \multicolumn{4}{|l|}{ Co-morbidities (diagnosis and/or treatment): } \\
\hline Diabetes, $\mathrm{N}(\%)$ & $8(11 \%)$ & $17(4 \%)$ & 0.04 \\
\hline Hyperlipidemia, $\mathrm{N}(\%)$ & $7(9 \%)$ & $27(7 \%)$ & 0.43 \\
\hline High Blood Pressure, N (\%) & $5(7 \%)$ & $27(7 \%)$ & 0.97 \\
\hline \multicolumn{4}{|l|}{ Laboratory values, mean \pm SD: } \\
\hline \multicolumn{4}{|l|}{ Liver panel and platelets: } \\
\hline $\operatorname{ALT}(\mathrm{U} / \mathrm{L})$ & $37.4 \pm 6.8$ & $123.1 \pm 87.6$ & $<0.00001$ \\
\hline AST (U/L) & $31.6 \pm 7.8$ & $71.9 \pm 48.4$ & $<0.00001$ \\
\hline GGT (U/L) & $29.8 \pm 20.2$ & $50.7 \pm 36.2$ & $<0.00001$ \\
\hline Creatinine (mg/dL) & $0.58 \pm 0.14$ & $0.63 \pm 0.58$ & 0.89 \\
\hline Bilirubin, direct (mg/dL) & $0.09 \pm 0.05$ & $0.09 \pm 0.07$ & 0.54 \\
\hline Albumin (g/dL) & $4.4 \pm 0.4$ & $4.5 \pm 0.4$ & 0.32 \\
\hline Internationalized normalized ratio (INR) & $1.02 \pm 0.07$ & $1.04 \pm 0.07$ & 0.12 \\
\hline Platelets (1000 cells/mcL) & $301.4 \pm 69.4$ & $292.6 \pm 70.8$ & 0.31 \\
\hline \multicolumn{4}{|l|}{ Fasting lipid profile: } \\
\hline Triglycerides (mg/dL) & $143.1 \pm 86.0$ & $147.7 \pm 87.0$ & 0.73 \\
\hline Cholesterol, total (mg/dL) & $164.9 \pm 32.6$ & $171.0 \pm 39.2$ & 0.24 \\
\hline Cholesterol, HDL (mg/dL) & $39.3 \pm 8.9$ & $38.5 \pm 9.7$ & 0.41 \\
\hline Cholesterol, LDL (mg/dL) & $97.8 \pm 24.6$ & $103.2 \pm 30.5$ & 0.16 \\
\hline \multicolumn{4}{|l|}{ Fasting glucose and insulin: } \\
\hline Glucose (mg/dL) & $87.0 \pm 21.0$ & $88.5 \pm 16.6$ & 0.03 \\
\hline Insulin (mg/dL) & $29.4 \pm 19.9$ & $35.4 \pm 37.6$ & 0.27 \\
\hline HbA1c (\%) & $5.4 \pm 0.6$ & $5.4 \pm 0.7$ & 0.47 \\
\hline
\end{tabular}




\begin{tabular}{|c|c|c|c|}
\hline & \multicolumn{3}{|c|}{ ALT level $(\mathbf{U} / \mathbf{L})^{*}$} \\
\hline & $\begin{array}{r}\text { Mildly } \\
\text { Elevated } \\
(N=74)\end{array}$ & $\begin{array}{r}\text { Elevated } \\
(\mathbf{N}=392)\end{array}$ & $\mathbf{P}^{\dagger}$ \\
\hline HOMA-IR & $6.3 \pm 4.4$ & $7.9 \pm 9.1$ & 0.19 \\
\hline \multicolumn{4}{|l|}{ Histologic characteristics, $N(\%)$ : } \\
\hline Steatosis grade: & & & $<0.0001$ \\
\hline $0-4 \%$ & $5(7 \%)$ & $11(3 \%)$ & \\
\hline $5-33 \%$ (mild) & $32(43 \%)$ & $89(23 \%)$ & \\
\hline$>33 \%$ (moderate or severe) & $37(50 \%)$ & $292(74 \%)$ & \\
\hline Lobular inflammation, $20 \times$ mag: & & & $<0.0001$ \\
\hline$<2$ & $59(80 \%)$ & $203(52 \%)$ & \\
\hline$\geq 2$ & $15(20 \%)$ & $189(48 \%)$ & \\
\hline Portal chronic inflammation: & & & 0.003 \\
\hline None & $13(18 \%)$ & $27(7 \%)$ & \\
\hline Mild/More than mild & $61(82 \%)$ & $365(93 \%)$ & \\
\hline Ballooning injury: & & & 0.006 \\
\hline None & $51(69 \%)$ & $202(52 \%)$ & \\
\hline Few/many & $23(31 \%)$ & $190(48 \%)$ & \\
\hline Acidophil bodies: & & & 0.003 \\
\hline Rare/absent & $53(72 \%)$ & $206(53 \%)$ & \\
\hline Many & $21(28 \%)$ & $186(47 \%)$ & \\
\hline Fibrosis stage: & & & 0.001 \\
\hline None $(0)$ & $34(46 \%)$ & $106(27 \%)$ & \\
\hline \multicolumn{4}{|l|}{ Mild: (1) } \\
\hline Perisinusoidal, Zone $3,(1 \mathrm{a})^{\wedge}$ & $6(8 \%)$ & $27(7 \%)$ & \\
\hline Perisinusoidal, Zone $3,(1 \mathrm{~b})^{\wedge}$ & $1(1 \%)$ & $17(4 \%)$ & \\
\hline Portal, periportal (1c) & $21(28 \%)$ & $113(29 \%)$ & \\
\hline \multicolumn{4}{|l|}{ Moderate (2): } \\
\hline Perisinusoidal and portal/periportal & $5(7 \%)$ & $70(18 \%)$ & \\
\hline \multicolumn{4}{|l|}{ Severe $(3$ or 4$)$} \\
\hline Bridging or Cirrhosis $(3,4)$ & $7(9 \%)$ & $57(15 \%)$ & \\
\hline Steatohepatitis diagnosis: & & & $<0.0001$ \\
\hline NAFLD, not NASH & $40(54 \%)$ & $89(23 \%)$ & \\
\hline Suspicious/borderline Zone 3 (1a) & $13(18 \%)$ & $72(18 \%)$ & \\
\hline Suspicious/borderline Zone 1 (1b) & $14(19 \%)$ & $115(29 \%)$ & \\
\hline Definite & $7(9 \%)$ & $116(30 \%)$ & \\
\hline NAFLD Activity Score: & & & $<0.0001$ \\
\hline $0-3$ & $47(64 \%)$ & $111(28 \%)$ & \\
\hline $4-8$ & $27(36 \%)$ & $281(72 \%)$ & \\
\hline Mean \pm SD & $3.2 \pm 1.2$ & $4.4 \pm 1.5$ & $<0.00001$ \\
\hline
\end{tabular}

J Pediatr. Author manuscript; available in PMC 2015 April 01. 
No. missing values for a characteristic reported in parentheses by ALT group $(26-50,>50)$ : Caucasian $(0,1)$, Hispanic $(1,0)$, Triglycerides $(0,4)$, Cholesterol: total $(0,3)$, HDL $(0,4)$, LDL (1,6), Glucose $(0,2)$, Insulin, HOMA-IR $(5,1), \operatorname{HbA1c}(0,5)$, Bilirubin $(0,1)$, INR $(1,1)$, Fibrosis $(0,2)$

${ }^{\dagger} \mathrm{P}$ values (2-sided) determined from either a Fisher exact test, a chi-square test or a Cochran-Armitage test for trend (for ordered categories) for categorical variables or a Wilcoxon Rank sum test for continuous variables.

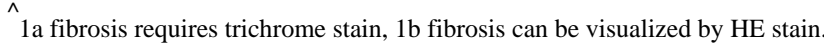

